\title{
Protocol for developing clinical practice guidelines on traditional Chinese medicine therapy for influenza
}

\author{
Guozhen Zhao, ${ }^{1,2}$, Yuhong Guo ${ }^{1}$, Wei Chen ${ }^{2}$, Bo Li ${ }^{1}$, Tengfei Chen ${ }^{1}$, Youran Lu ${ }^{1}$, Yumeng Yan $^{1,2}$, \\ Yafan Wang ${ }^{1}$, Qingquan Liu ${ }^{1}$ \\ ${ }^{1}$ Beijing Hospital of Traditional Chinese Medicine, Capital Medical University, Beijing, China; ${ }^{2}$ Beijing University of Chinese Medicine, Beijing, \\ China \\ Correspondence to: Qingquan Liu. Beijing Hospital of Traditional Chinese Medicine, No. 23 Meishuguanhoujie, Dongcheng District, Beijing, China. \\ Email: liuqingquan2003@126.com.
}

\begin{abstract}
Background Influenza is the most prevalent acute respiratory infection worldwide. There are many different traditional Chinese medicine (TCM) therapies, which could reduce the duration of fever during influenza. However, there are no clinical practice guidelines (CPG) involving TCM therapies for influenza. Therefore, the present study aimed to establish a protocol for the development of CPG on TCM therapy for influenza.

Methods: The CPG will be developed according to the Institute of Medicine, the Appraisal of Guidelines for Research and Evaluation II, and the World Health Organization (WHO) guideline handbook, and will provide recommendations based on systematic reviews. We have established a guideline working group (including a guideline steering group, a guideline development group, a guideline secretary group, and a system evaluation group), and will formulate clinical questions based on the population, intervention, comparison, and outcomes format. The recommendations will be formed via evidence search, syntheses, and the nominal group technique method. We will also consider patients' values or preferences, peer review results, and declarations of interest in the CPG. The CPG is registered on the International Practice Guidelines Registry Platform (registration no. IPGRP-2019CN044).

Results: The protocol will provide a roadmap to develop an evidence-based CPG for influenza treated by TCM systematically. These guidelines would be the first CPG on TCM therapy for influenza, based on the WHO Handbook for Guideline Development, and will provide the necessary evidence for treating influenza using TCM.
\end{abstract}

Keywords: Influenza; traditional Chinese medicine (TCM); clinical practice guidelines (CPG); protocol; World Health Organization (WHO)

Submitted Mar 31, 2020. Accepted for publication Aug 26, 2020.

doi: 10.21037/apm-20-862

View this article at: http://dx.doi.org/10.21037/apm-20-862

\section{Introduction}

\section{Description of disease}

Influenza is an acute respiratory infection caused by influenza viruses. Influenza is prone to mutations and spreads rapidly, causing seasonal epidemics. Most people recover from influenza (1); however, influenza can cause complications that result in severe illness and death, particularly in infants, the elderly, pregnant women, and patients with chronic conditions (2-5). Severe seasonal influenza affects $3-5$ million people worldwide and causes 290,000-650,000 deaths annually (6).

According to the viral nucleoprotein and matrix protein, influenza is classified into four types of viruses: A, B, C, and D (7). Influenza A viruses can be divided into a variety of subtypes based on the protein structure and genetic characteristics of hemagglutinin and neuraminidase distributed on the surface of the virus. Currently, 18 
subtypes of hemagglutinin (H1-18) and 11 subtypes of neuraminidase (N1-11) have been detected (8). Because the replication of influenza viruses is not involved in the RNAcorrecting enzyme, the RNA polymerase will make errors after about every 10,000 nucleotide replications (9), causing more frequent mutations than other viruses. Because of the variability of influenza virus genes and the diverse biologic characteristics of the host, new variant strains are continually emerging, so they can be widely spread in the population, causing recurrent infections and morbidity (10).

The main clinical manifestations of influenza are acute onset, fever (often up to $39-40{ }^{\circ} \mathrm{C}$ ), aversion to cold, chills, headache, muscle and joint pain, extreme fatigue, and loss of appetite. Other symptoms include sore throat, cough, nasal congestion, runny nose, post-sternal discomfort, facial flushing, mild conjunctival congestion, vomiting, and diarrhea (11). Pneumonia is the most common complication of influenza. Influenza patients with pneumonia, or persistent high fever, dyspnea, altered mental status, and dehydration, are considered critical, as this can lead to respiratory failure, acute necrotizing encephalopathy, septic shock, and multiple organ dysfunction (7). Therefore, the purpose of treating influenza is to shorten the time of fever, improve systemic symptoms, and reduce the incidence of severe and critical conditions.

\section{Description of interventions}

Many guidelines have considered the prevention and Western medical treatments of influenza, which mainly include prevention by vaccination injection and isolation, as well as the oral administration of M2 channel blockers, such as amantadine and rimantadine; neuraminidase inhibitors, such as zanamivir; and oseltamivir for antiviral treatment.

Traditional Chinese medicine (TCM) has certain advantages in the treatment of influenza. In a multicenter, randomized controlled trial, 410 patients with mild influenza A were randomly assigned to the control group, the oseltamivir group, the TCM group (modified Maxingshigan-Yinqiaosan decoction), and the oseltamivir + TCM group. The results showed that the length of time of the fever in the control group, the oseltamivir group, TCM group, and oseltamivir + TCM group was 26, 20, 16 , and 15 hours, respectively. It was concluded that TCM decoction could reduce the duration of fever, and its efficacy was similar to that of oseltamivir or superior. For patients with mild influenza, Maxingshigan-Yinqiaosan decoction as a compound formula in TCM is better than that of oseltamivir concerning the abatement of fever (12). Unfortunately, there are no guidelines for TCM in the treatment of influenza.

\section{Principles of TCM}

In TCM, it is believed that flu pathogenesis is through the invasion of the body by epidemic evils. If the evil invades the qi level, this is a mild case; if the evil blocks the lungs, it is considered a critical condition. In the diagnosis and treatment of TCM, syndrome differentiation is of significance in diagnosis, and the primary treatment strategy is to resolve toxins and vent evils out. Compared with antiviral drugs or corticosteroids for the treatment of influenza, TCM compound formulas are aimed at the struggle between the body and the pathogenic evils and venting the evils out of the body by the guidance of TCM theory. The timeframe of medication administration does not limit TCM treatment. TCM treatment does not differentiate between virus types and can reduce the incidence of drug resistance and severe complications caused by the misuse of antibiotics.

\section{Syndrome differentiation of influenza in TCM}

Syndrome differentiation is one of the characteristics of TCM, and our clinical practice guidelines (CPG) will classify influenza into five syndromes: (I) defensive qi invaded by wind and heat pattern. Clinical manifestations include fever (or no fever initially), inflammation of the pharynx and discomfort, light cough, minimal phlegm, absence of sweating, red tongue with a thin or greasy coating, and a floating and rapid pulse; (II) pulmonary invasion of heat and toxin pattern. Clinical manifestations include high fever, cough, sticky phlegm, difficulty coughing, thirst, sore throat, red eyes, red tongue with a yellow and slimy coating, and a slippery and rapid pulse; (III) pulmonary obstruction of heat and toxin pattern. Clinical manifestations include persistent high fever, severe cough, little or no phlegm, rapid wheezing and shortness of qi, headache and body pain with/without palpitations, restlessness, red tongue with a thin yellow or greasy coating, and a wiry and rapid pulse; (IV) internal fall of toxin and heat, inner blocking, and outer collapse pattern. Clinical manifestations include loss of consciousness, stagnation, dark purple lips and nails, shortness of breath, burning of chest and abdomen, cold hands and feet, less urination, dark red tongue, and a deep thread and rapid pulse; and (V) Qi 
and Yin deficiency, non-recovery of right qi pattern. Clinical manifestation includes spirit lassitude and lack of strength, shortness of breath, cough, less phlegm, poor appetite, dark or reddish tongue with a thin and greasy coating, and a wiry and thread pulse.

\section{Importance of developing CPG on TCM for influenza}

Currently, there re are no specific guidelines for the treatment of influenza using TCM. Therefore, we aimed to search and evaluate the existing evidence comprehensively and consider clinical physicians' experiences to develop CPG for influenza treated by TCM and to provide relevant evidence-based recommendations for clinicians, nurses, and patients.

\section{Objective}

The protocol will provide a roadmap to develop evidencebased CPG for influenza treated by TCM systematically. These guidelines would be the first CPG on TCM therapy for influenza, based on the World Health Organization (WHO)'s guideline development (13), and will provide substantial evidence for treating influenza using TCM.

\section{Methods}

\section{Principles}

Following the steps in the WHO Handbook for Guideline Development, the guidelines will be based on the latest guideline definition of the Institute of Medicine (IOM) and will follow The Grading of Recommendations Assessment, Development and Evaluation (GRADE) tool to form recommendations (13-16). The guidelines are registered on the International Practice Guide registration platform (no. IPGRP-2019CN044; http://www.guidelines-registry.org/).

\section{Guideline development institutions, target users, and population}

The guidelines will be jointly developed by the China Association of Chinese Medicine, Beijing Hospital of Traditional Chinese Medicine Affiliated to Capital Medical University, and Beijing University of Chinese Medicine. The methodology was supported by the Center for Epidemiology and Evidence-Based Medicine of Beijing University of Chinese Medicine. The target users of the guidelines are medical practitioners, nursing staff, and pharmacists in emergency departments or intensive care units. The target population is adult patients clinically or diagnosed with influenza.

\section{Guideline working group}

The guideline working group was established in June 2019 and consisted of four groups: the guideline steering group, the guideline development group, the guideline secretary group, and the systematic evaluation group. In total, there are 38 members from different disciplines: twenty-nine experts in clinic, four in evidence-based medicine, one in health economics, two in pharmacy, and two in nursing. The 38 members come from eight provinces in China, covering China's northeast, northwest, north, southwest, south, and other regions.

Four members of the guideline steering group are mainly responsible for: (I) drafting the scope of the guidelines and forming critical questions following the population, intervention, comparison, and outcome (PICO) format; (II) selecting the system evaluation team and the guideline methodologist; (III) selecting the members of the guideline development group and external review members; (IV) identifying the guideline plan; (V) monitoring the evidence search, evaluation, and synthesis; (VI) managing and assessing conflicts of interest; (VII) organizing guideline development meetings; (VIII) collaborating with authors to draft guidelines, monitoring peer reviews, and revising the guideline draft appropriately; and (IX) overseeing the publication and promotion of the guidelines, and monitoring and evaluating the update of the guidelines.

The guideline development group is comprised of 24 members, whose primary responsibilities are: (I) providing advice on determining the scope of the guideline; (II) assisting the guideline steering group to formulate critical questions, following the PICO format; (III) ranking the outcome indicators following their significance; (IV) appraising the evidence levels and recommendation strength using the GRADE tool; (V) drafting the guidelines; and (VI) publishing and promoting the guidelines.

The guideline secretary group is composed of three members, whose primary responsibilities are: (I) assisting the guideline steering group in the development of the guidelines; (II) recording the development of guidelines in details; and (III) communicating with and contacting the members of the guideline working group.

The system evaluation group is composed of seven 
members, whose primary responsibilities are: (I) conducting evidence retrieval and synthesis for each recommendation; and (II) formulating a summary table of GRADE evidence.

\section{Declaration of interests and funding support}

All members of the guideline development group, the guideline steering group, the guideline secretary, and the system evaluation group will be required to state their conflicts of interest before attending the guideline meetings.

\section{Formulating questions and choosing outcomes}

Based on interviews with two academicians in the emergency field in the guideline steering group, the preliminary clinical issues of these guidelines were formulated. This will follow with a guideline development group meeting, where these clinical questions will be finally determined using the nominal group technique (NGT). The format of the clinical questions should follow the PICO principle. For example:

Compared with oseltamivir treatment, are Finhua Qinggan granules better at shortening fever time in patients with influenza $A$ ?

Here, "P" indicates all patients identified as diagnosed with influenza A, "I" indicates patients taking Jinhua Qinggan granules, "C" indicates patients receiving oseltamivir treatment, and "O" indicates the fever time.

Based on the preliminary analysis of previous literature and past clinical practice, the following outcome indicators were selected in this protocol, including symptom relief time, fever abatement time, and recovery time. Each outcome indicator can represent the efficacy of the treatment of influenza. Methodological experts are responsible for organizing clinical doctors to produce a list of outcomes and to grade and rank them. According to the 9-point scale, 1-3 points indicate non-importance of the outcomes, 4-6 points indicate the importance of the outcomes and 7-9 points indicate the extreme importance of the outcomes.

\section{Evidence retrieval and synthesis}

\section{Databases}

Databases, such as Medline, Embase, the Cochrane Library, as well as four databases in the Chinese language, including Sinomed, CNKI, VIP, and WanFang, will be searched. The search deadline is August in 2020.

\section{Search terms}

The main search terms include flu, influenza, influenza A, influenza B, Chinese medicine, and Chinese herbal medicine. We will first search the MeSH terms in the Medline database to determine the subject terms and free words. Based on this, the evidence-based medical experts will provide guidance and develop a detailed search strategy.

\section{Literature selection}

Systematic reviews, randomized controlled trials (RCTs), and other types of clinical studies related to the study will be retrieved. After removing duplicates, the guideline working group will screen the included literature based on the title and abstract, and then repeat this according to the full text to determine the final included literature.

\section{Evidence syntheses}

High-quality systematic reviews published in the past 2 years will be adopted in the study. The systematic evaluation group will also develop a new systematic review based on the best evidence available now for evidence synthesis.

\section{Evidence assessment}

The GRADE tool will be used to evaluate the level of evidence that will be used to formulate recommendations. The systematic evaluation group will develop a summary table of evidence, and the guideline development group will classify the relevant evidence for each recommendation into four levels based on the GRADE tool: high, moderate, low, and very low. RCTs are considered high-level evidence, whereas observational studies are considered lowlevel evidence. Clinical experts and methodologists will jointly adjust the level of evidence according to the five downgrading criteria and three upgrading criteria of the GRADE tool to form the final version.

\section{Develop recommendation strength}

After completing the evidence summary form, the guideline development group will discuss the quality of the evidence, the patients' values and preferences, the advantages and disadvantages, and the impact of resources according to the GRADE tool. It will determine the strength of each recommendation using the NGT.

\section{Patients' values and preferences}

The guideline secretary will be responsible for investigating 
the values and hospitals using TCM to treat patients with influenza. Patients' opinions will be fed back to the guideline steering group and the guideline development group for consideration in the development of recommendations.

\section{Drafting the guidelines}

The guideline writer and the guideline steering group will draft the guidelines collaboratively. The guidelines will be drafted using the evidence summary for each recommendation, the level of evidence, and the strength of the recommendation. The draft guidelines should abide by the requirements of the CPGs issued by the China Association of Chinese Medicine (CACM).

\section{Peer review of the guidelines}

The draft guidelines will be peer reviewed by external experts. The guideline development group needs to record the review process and collect the reviewers' proposals. After a full discussion of these comments, the draft guidelines can be appropriately revised if necessary.

\section{Publication and updating of the guidelines}

The guidelines will be published in the form of a group standard form of the CACM within 1 year. The guidelines will be translated into English and published in relevant journals. The guideline development group will develop patient guidelines targeting patients as needed. The guideline development group will update the guidelines 25 years, with the consideration of specific conditions. The factors initiating the guideline update process include the emergence of new relevant evidence after the publication of the guidelines, evidence changes affecting the guideline recommendations, and changes in the strength of the guideline recommendations.

\section{Promotion, implementation, and evaluation of the guidelines}

After issuing the guidelines, the CACM will promote it as follows: (I) a guideline issuing ceremony will be held by the Emergency Branch of the CACM; (II) the guidelines will be printed as a manual and disseminated in academia; (III) the content of the guidelines will be used in lectures in academic conferences; and (IV) the full text of the guidelines will be promoted on the Internet.

\section{Acknowledgments}

Funding: The present study was supported by the National Science and Technology Major Project (No. 2017ZX10305501001).

\section{Footnote}

Provenance and Peer Review: This article was commissioned by the editorial office, Annals of Palliative Medicine for the series "Narrative \& Evidence-based Medicine for Traditional Medicine: from basic research to clinical practice and trial". The article has undergone external peer review.

Conflicts of Interest: All authors have completed the ICMJE uniform disclosure form. available at http://dx.doi. org/10.21037/apm-20-862. The series "Narrative \& Evidence-based Medicine for Traditional Medicine: from basic research to clinical practice and trial" was commissioned by the editorial office without any funding or sponsorship. BL served as the unpaid Guest Editor of the series. The authors have no other conflicts of interest to declare.

Ethical Statement: The authors are accountable for all aspects of the work in ensuring that questions related to the accuracy or integrity of any part of the work are appropriately investigated and resolved.

Open Access Statement: This is an Open Access article distributed in accordance with the Creative Commons Attribution-NonCommercial-NoDerivs 4.0 International License (CC BY-NC-ND 4.0), which permits the noncommercial replication and distribution of the article with the strict proviso that no changes or edits are made and the original work is properly cited (including links to both the formal publication through the relevant DOI and the license). See: https://creativecommons.org/licenses/by-nc-nd/4.0/.

\section{References}

1. Uyeki TM, Bernstein HH, Bradley JS, et al. Clinical Practice Guidelines by the Infectious Diseases Society 
of America: 2018 Update on Diagnosis, Treatment, Chemoprophylaxis, and Institutional Outbreak Management of Seasonal Influenzaa. Clin Infect Dis 2019;68:895-902.

2. Poehling KA, Edwards KM, Weinberg GA, et al. The underrecognized burden of influenza in young children. $\mathrm{N}$ Engl J Med 2006;355:31-40.

3. Siston AM, Rasmussen SA, Honein MA, et al. Pandemic 2009 influenza A(H1N1) virus illness among pregnant women in the United States. JAMA 2010;303:1517-25.

4. Zhou H, Thompson WW, Viboud CG, et al. Hospitalizations associated with influenza and respiratory syncytial virus in the United States, 1993-2008. Clin Infect Dis 2012;54:1427-36.

5. Thompson WW, Moore MR, Weintraub E, et al. Estimating influenza-associated deaths in the United States. Am J Public Health 2009;99 Suppl 2:S225-30.

6. World Health Organization. Fact sheet on influenza (seasonal). Available online: https://www.who.int/en/newsroom/fact-sheets/detail/influenza-(seasonal)

7. National Health and Family Planning Commission of the People's Republic of China. Influenza treatment plan (2018 edition). Chinese Journal of Clinical Infectious Diseases 2018;(1):1-5.

8. US CDC. Types of Influenza Viruses. Available online: https://www.cdc.gov/flu/about/viruses/types.htm

Cite this article as: Zhao G, Guo Y, Chen W, Li B, Chen T, Lu Y, Yan Y, Wang Y, Liu Q. Protocol for developing clinical practice guidelines on traditional Chinese medicine therapy for influenza. Ann Palliat Med 2021;10(12):13024-13029. doi: 10.21037/ apm-20-862
9. Drake JW. Rates of spontaneous mutation among RNA viruses. Proc Natl Acad Sci USA 1993;90:4171-5.

10. Vaccines against influenza WHO position paper November 2012. Wkly Epidemiol Rec 2012;87:461-76.

11. Influenza diagnosis and treatment guidelines. Beijing: Ministry of Health of the People's Republic of China, 2011.

12. Wang C, Cao B, Liu QQ, et al. Oseltamivir compared with the Chinese traditional therapy maxingshigan-yinqiaosan in the treatment of H1N1 influenza: a randomized trial. Ann Intern Med 2011;155:217-25.

13. World Health Organization. WHO Handbook of Guideline Development 2nd edition. Available online: http://www.who.int/kms/handbook_2nd_ed.pdf/

14. Kung J, Miller RR, Mackowiak PA. Failure of clinical practice guidelines to meet institute of medicine standards: Two more decades of little, if any, progress. Arch Intern Med 2012;172:1628-33.

15. The AGREE Reporting Checklist: a tool to improve reporting of clinical practice guidelines, BMJ 2016;354:i4852.

16. Guyatt GH, Oxman AD, Vist GE, et al. GRADE: an emerging consensus on rating quality of evidence and strength of recommendations. BMJ 2008;336:924-6.

(English Language Editors: R. Scott and J. Chapnick) 